\title{
ERRATUM: CYLINDRICAL OPTIMAL REARRANGEMENT PROBLEM LEADING TO A NEW TYPE OBSTACLE PROBLEM
}

\author{
HAYK MikAYELYAN*
}

\begin{abstract}
A mistake in the proof of the Theorem 5.5 on page 870 in [H. Mikayelyan, ESAIM: COCV
\end{abstract} 24 (2018) 859-872] has been found.

Mathematics Subject Classification. 35R35, 49J20.

Received March 31, 2021. Accepted April 3, 2021.

ESAIM: COCV 24 (2018) 859-872, https://doi.org/10.1051/cocv/2017047

The proof of the Theorem 5.5 on page 870 in [1] (below) contains a mistake.

Theorem 1.1. Let $\Omega=D \times(0,1), I(u)\left(x^{\prime}\right)=\int_{0}^{1} u\left(x^{\prime}, x_{n}\right) \mathrm{d} x_{n}$, and $u_{1}$ and $u_{2}$ minimize

$$
J(u)=\int_{\Omega}|\nabla u|^{2}+\chi_{\{I(u)>0\}} u \mathrm{~d} x,
$$

among $W^{1,2}(\Omega)$ functions with constant boundary data $\alpha_{1}$ and $\alpha_{2}$ respectively, where $0<\alpha_{1}<\alpha_{2}$. Then

$$
I\left(u_{1}\right)\left(x^{\prime}\right) \leq I\left(u_{2}\right)\left(x^{\prime}\right)
$$

for $x^{\prime} \in D$.

In the Step 1 of the proof the following argument has been applied:

For $\delta \geq 0$ let $u_{\delta}$ be the minimizer of the convex functional

$$
J_{\delta}(u)=\int_{\Omega}|\nabla u|^{2}+\chi_{\{I(u)>\delta\}} u \mathrm{~d} x,
$$

among the functions $u \in W^{1,2}(\Omega)$ with boundary values $u=\alpha_{2}$. Let us prove that $u_{2} \leq u_{\delta}$.

Keywords and phrases: Obstacle problem, rearrangements.

School of Mathematical Sciences, University of Nottingham Ningbo China, 199 Taikang East Road, Ningbo 315100, Zhejiang Prov., PR China.

* Corresponding author: hayk.mikayelyan@nottingham.edu.cn 
Assume $\tilde{\Omega}=\left\{x \mid u_{2}(x)<u_{\delta}(x)\right\} \neq \emptyset$ and set $u_{3}=\min \left(u_{2}, u_{\delta}\right)$. If $\int_{\tilde{\Omega}}\left|\nabla u_{2}\right|^{2} \mathrm{~d} x<\int_{\tilde{\Omega}}\left|\nabla u_{\delta}\right|^{2} \mathrm{~d} x$ then

$$
J_{\delta}\left(u_{3}\right)<J_{\delta}\left(u_{\delta}\right)
$$

Otherwise if $\int_{\tilde{\Omega}}\left|\nabla u_{2}\right|^{2} \mathrm{~d} x \geq \int_{\tilde{\Omega}}\left|\nabla u_{\delta}\right|^{2} \mathrm{~d} x$ then

$$
J\left(u_{3}\right)<J\left(u_{2}\right) .
$$

Equations (1.1) and (1.2) contradict the fact that $u_{\delta}$ and $u_{2}$ are minimizers.

The equation (1.2) is not necessarily true, and the whole proof does not work. If we would "fix" the definition of $\tilde{\Omega}$ to $\tilde{\Omega}=\left\{x \mid u_{2}(x)>u_{\delta}(x)\right\} \neq \emptyset$, the argument would fail at equation (1.1).

All other results in [1] are unaffected by this and are correct.

It is an open question whether the Theorem 1.1 is correct or not. Some numerical experiments seem to confirm the statement.

The author is grateful to Zhiwei Cheng for pointing out the mistake.

\section{REFERENCE}

[1] H. Mikayelyan, Cylindrical optimal rearrangement problem leading to a new type obstacle problem. ESAIM: COCV 24 (2018) 859-872. 